\title{
Inverse QRD BEACON Algorithm
}

\author{
José A. Apolinário Jr., Mohammad Mobien Shoaib, and Stefan Werner
}

\begin{abstract}
Resumo-Este artigo deriva a versão usando decomposição QR inversa (IQRD) do algoritmo Bounding Ellipsoidal Adaptive CONstrained least-squares (BEACON). O algoritmo BEACON pertence à família de algoritmos conhecida como set-membership filtering (SMF) que apresenta atualização esparsa no tempo e boa capacidade de rastreamento. A característica proeminente de atualização esparsa em SMF vem de uma restrição predefinida quanto aos limites do erro de saída especificada no projeto do filtro. Como uma conseqüiência, um conjunto de estimativas válidas de vetores de coeficientes se conformarão à restrição ao invés de uma estimativa pontual. A escolha da restrição ao erro aparece naturalmente em várias aplicações de processamento de sinais, por exemplo, quando a ordem do modelo não é conhecida ou a distância entre pontos de uma constelação é conhecida a priori num equalizador do tipo decision-feedback. O novo algoritmo, o IQRD-BEACON, implementa a mesma função objetivo que o BRACON e, portanto, apresentará, em precisão infinita, resultados idênticos em termos de curvas de aprendizagem e freqüência de atualização. A vantagem do IQRDBEACON vem com o uso de rotações numericamente estáveis nas equações de atualização, evitando pois o uso de recursões malcondicionadas associadas ao emprego do lema de inversão de matrizes no BEACON convencional. Nossas reivindicações com respeito ao desempenho do IQRD-BEACON são verificadas por meio de simulações em computador.
\end{abstract}

Palavras-Chave-Filtragem adaptativa, Filtragem SetMembership filtering, Algoritmos com decomposição QR.

Abstract-This paper derives the inverse QR-decomposition (IQRD) version of the Bounding Ellipsoidal Adaptive CONstrained least-squares (BEACON) algorithm. The BEACON algorithm belongs to the family of set-membership filtering (SMF) algorithms that feature sparse updating in time and good tracking capability. The prominent characteristic of sparse updating in SMF arises from a predefined bounded error-constraint specified in the filter design. As a consequence, a set of valid coefficient vector estimates will conform to the constraint rather than a single point-estimate. The choice of the error constraint appears naturally in various signal processing applications, e.g., when model-order is unknown or distance between constellation points is a priori known in a decision-feedback equalizer. The new algorithm, the IQRD-BEACON, implements the same objective function as BEACON and will, therefore, in infinite-precision environment present identical results in terms of learning curves and update frequency. The advantage of the IQRD-BEACON comes with the use of numerically stable rotations in the update equations, thus avoiding the use of the ill-conditioned recursions associated with the matrix-inversion lemma employed in the conventional BEACON. Our claims regarding the performance of the IQRD-BEACON are verified through computer simulations.

Keywords-Adaptive filtering, Set membership filtering, QRDRLS algorithm.

J. A. Apolinário Jr., Departamento de Engenharia Elétrica, Instituto Militar de Engenharia, Rio de Janeiro, Brazil, E-mail: apolin@ieee. org, Mobien Shoaib and Stefan Werner, Signal Processing Laboratory, Helsinki University of Technology, Espoo, Finland, E-mails: mobien.shoaibetkk.fi and stefan.wernerdtkk. fi.

\section{INTRODUCTION}

Set-membership filtering (SMF) algorithms may be considered attractive options for a wide range of adaptive filtering applications. This is due to their reduced average computational complexity when compared to their conventional LMS and RLS counterparts. In addition, they feature fast convergence and good tracking capability. In SMF algorithms, coefficientvector updating is not performed unless the output error of the filter is larger than a certain threshold. This sparse updating in time (or data-selectivity) enables efficient usage of shared resources when multiple adaptation processes are handled simultaneously, or reduced power consumption is desired.

The SMF concept has been successfully employed in a number of algorithms that minimize the MSE (Mean Squared Error) by changing the respective objective function such that a bound is specified on the magnitude of error, e.g., Setmembership Normalized Least Mean Square (SM-NLMS) [1] and the Set-membership Affine Projection Algorithm (SMAPA) [2]. The same idea can also be extended to another objective function including the Weighted Least Squares used by the RLS (Recursive Least Squares) family of algorithms [3].

In [4], a recursive algorithm named BEACON was derived according to an optimal bounding ellipsoid (OBE) criterion. The BEACON algorithm was shown to feature a highly selective update mechanism (approximately 5\% of the time) and an ability to track fast time-varying conditions.

Although other OBE algorithms were implemented using Givens rotations [3], [5], [4], this paper implements a QR decomposition version of the BEACON [4] algorithm based on the inverse Cholesky factor.

This paper is organized as follows: in Section II, basic concepts concerning the SMF algorithms are reviewed as well as the basic derivation of the BEACON algorithm. The inverse (and the basic equations for a direct) QRD-WLS version of the BEACON algorithm is derived in Section III. Simulation results are detailed in Section IV and conclusions are summarized in Section V.

\section{Set MEMbership FILtering AND the BEACON}

Mean-square error (MSE) based adaptive filtering algorithms such as the Least-Mean-Square (LMS) algorithm or the Affine Projection Algorithm (APA) [6] search, at time instant $k$, a coefficient vector $\boldsymbol{w}$ that minimizes $E\left[e^{2}(k)\right]$, where the output estimation error is given by

$$
e(k)=d(k)-\boldsymbol{w}^{T} \boldsymbol{x}(k)
$$

with $d(k)$ being the reference signal and $\boldsymbol{x}(k)$ the input-signal vector.

In set-membership filtering (SMF), an upper bound of the output estimation error is specified such that all coefficient 
vectors satisfying the error constraint are considered feasible. The resulting adaptation algorithms are data-selective with a considerably reduced average computational complexity.

As an example, the Set-Membership Normalized LMS (SMNLMS) algorithm proposed in [1], updates the coefficient vector $\boldsymbol{w}(k-1)$ to $\boldsymbol{w}(k)$ only if the a priori output error exceeds a certain threshold $\gamma$. Let $\mathcal{S}$ denote the space model, i.e., $(\boldsymbol{x}, d) \in \mathcal{S}$, and $\Theta$ the set of all possible vectors $\boldsymbol{w}$ that result in an error with a norm not exceeding $\gamma$. The feasibility set $\Theta$ is defined as the set of all filter vectors $w$ satisfying the error constraint for all possible input-desired data pairs and is given by

$$
\Theta=\bigcap_{(\boldsymbol{x}, d) \in \mathcal{S}}\left\{\boldsymbol{w} \in \Re^{N}:\left|d-\boldsymbol{w}^{T} \boldsymbol{x}\right| \leq \gamma\right\}
$$

The set of all $\boldsymbol{w}$ satisfying the error bound, obtained after training with the $k$-th input-desired data pair $\{\boldsymbol{x}(k), d(k)\}$, denoted by $\mathcal{H}(k)$, is called the constraint set and can be expressed as

$$
\mathcal{H}(k)=\left\{\boldsymbol{w} \in \Re^{N}:\left|d(k)-\boldsymbol{w}^{T} \boldsymbol{x}(k)\right| \leq \gamma\right\}
$$

The exact membership set $\psi(k)=\cap_{i=0}^{k} \mathcal{H}(i)$ is the superset of the feasibility set and is defined as the minimal set estimate for $\Theta$ at time $k$. Also note that the feasibility set $\Theta$ lies in the constraint set $\mathcal{H}(k)$.

The objective is to estimate the membership set $\psi(k)$ at each instant $k$ in order to find the weights $\boldsymbol{w}$ satisfying the bound. The membership set $\psi(k)$ forms an $N$-dimensional convex polytope, which is not easily computed. The problem is greatly simplified if a tightly outer bounded ellipsoid $\varepsilon_{k}$ is estimated instead. The ellipsoid $\varepsilon_{k}$ is defined as

$$
\varepsilon_{k}=\left\{\boldsymbol{w} \in \Re^{N}:\left(\boldsymbol{w}-\hat{\boldsymbol{w}}_{k}\right)^{T} \boldsymbol{R}_{k}\left(\boldsymbol{w}-\hat{\boldsymbol{w}}_{k}\right) \leq \sigma_{k}\right\}
$$

where $\sigma_{k}>0$ and $\boldsymbol{R}_{k}$ is a deterministic weighted autocorrelation matrix of the input signal. Using this, it is possible to define an ellipsoid $\varepsilon_{0}$ as the set of all vector $\boldsymbol{w}$ such that $\left\{\boldsymbol{w} \in \Re^{N}:\left(\boldsymbol{w}-\hat{\boldsymbol{w}}_{0}\right)^{T} \boldsymbol{R}_{0}\left(\boldsymbol{w}-\hat{\boldsymbol{w}}_{0}\right) \leq \sigma_{0}\right\}$ where $\hat{\boldsymbol{w}}_{0}$ is the first estimate of $\boldsymbol{w}_{0}$ and $\boldsymbol{R}_{0}$ is the first estimate of $\boldsymbol{R}$.

Note that, if we initialize $\boldsymbol{R}_{0}=\boldsymbol{I}$, the ellipsoid $\varepsilon_{0}$ will actually become a circle. Moreover, for $k=1$, the ellipsoid $\varepsilon_{1}$ is shown as in Fig. 1 where $\varepsilon_{1} \supset\left\{\varepsilon_{0} \cap \mathcal{H}(1)\right\}$.

The basic idea of OBE algorithms, as seen in [4] is to outer bound the membership set at each instant by a mathematically tractable ellipsoid:

$$
\varepsilon_{k} \supset\left\{\varepsilon_{k-1} \cap \mathcal{H}(k)\right\} \supset \psi(k) .
$$

The process is carried out given an initial ellipsoid $\varepsilon_{0}=$ $\left\{\boldsymbol{w} \in \Re^{N}:\left(\boldsymbol{w}-\hat{\boldsymbol{w}}_{0}\right)^{T} \boldsymbol{S}^{-1}(0)\left(\boldsymbol{w}-\hat{\boldsymbol{w}}_{0}\right) \leq \sigma_{0}\right\}$ with some properly initialized estimates $\hat{\boldsymbol{w}}_{0}$ and $\boldsymbol{S}(0)=\boldsymbol{R}_{0}^{-1}$; the algorithm then starts a recursive procedure for computing the sequence of ellipsoids.

Assuming that we have, at time $k-1$, all data pairs $(\boldsymbol{x}(0), d(0))$ to $(\boldsymbol{x}(k-1), d(k-1))$, the updated coefficient vector of the BEACON algorithm is obtained from the minimization of a cost function $\boldsymbol{V}_{k-1}(\boldsymbol{w})=(\boldsymbol{w}-\boldsymbol{w}(k-$ 1) $)^{T} \boldsymbol{S}^{-1}(k-1)(\boldsymbol{w}-\boldsymbol{w}(k-1))-\sigma_{k-1}$ subject to $\mid d(k)-$ $\left.\boldsymbol{w}^{T} \boldsymbol{x}(k)\right|^{2} \leq \gamma^{2}$ which implies that $\boldsymbol{w}(k) \in \mathcal{H}(k)$. The

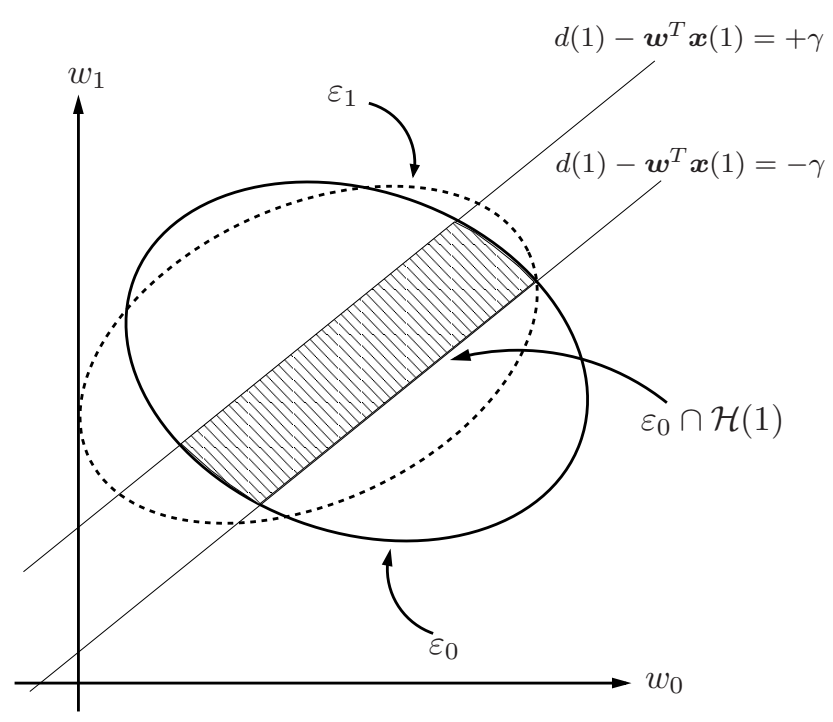

Fig. 1. First iteration of the OBE procedure.

recursions for the BEACON algorithm are quite similar in form with the equations of the conventional RLS algorithm and are presented in Table I, see [4] for the details of the

\begin{tabular}{|c|}
\hline BEACON \\
\hline $\begin{array}{l}\text { Initialize } \gamma, \boldsymbol{S}(-1)=\frac{1}{\hat{\sigma}_{x}^{2}} \boldsymbol{I}, \text { and } \boldsymbol{w}(-1) \\
\text { for } k=0,1, \ldots \\
\left\{\begin{array}{l}e(k)=d(k)-\boldsymbol{w}^{T}(k-1) \boldsymbol{x}(k) \\
\text { if }|e(k)| \leq \gamma \\
\text { then } \% \text { Do nothing: } \\
\quad \lambda_{k}=0 \Rightarrow\left\{\begin{array}{l}\boldsymbol{S}(k)=\boldsymbol{S}(k-1) \\
\boldsymbol{w}(k)=\boldsymbol{w}(k-1)\end{array}\right. \\
\text { else } \% \text { Update the BEACON Alg. : } \\
\quad \lambda_{k}=\frac{1}{\boldsymbol{x}^{T}(k) \boldsymbol{S}_{(k-1) \boldsymbol{x}(k)}\left(\frac{|e(k)|}{\gamma}-1\right)} \\
\quad \boldsymbol{\kappa}(k)=\frac{\lambda_{k} \boldsymbol{S}_{(k-1) \boldsymbol{x}(k)}}{1+\lambda_{k} \boldsymbol{x}^{T}(k) \boldsymbol{S}(k-1) \boldsymbol{x}(k)} \\
\quad \boldsymbol{S}(k)=\boldsymbol{S}(k-1)-\boldsymbol{\kappa}(k) \boldsymbol{x}^{T}(k) \boldsymbol{S}(k-1) \\
\boldsymbol{w}(k)=\boldsymbol{w}(k-1)+e(k) \boldsymbol{\kappa}(k)\end{array}\right.\end{array}$ \\
\hline
\end{tabular}
derivation.

TABEL I

THE BEACON ALGORITHM.

\section{THE INVERSE QRD-WLS BEACON}

Comparing the BEACON algorithm in Table I with the conventional RLS algorithm, it can be seen that its coefficient vector can be expressed as $\boldsymbol{w}(k)=\boldsymbol{S}(k) \boldsymbol{p}(k), \boldsymbol{S}(k)=$ $\boldsymbol{R}^{-1}(k)$ or

$$
\boldsymbol{w}(k)=[\underbrace{\sum_{i=0}^{k} \lambda_{i} \boldsymbol{x}(i) \boldsymbol{x}^{T}(i)}_{\boldsymbol{R}(k)}]^{-1} \underbrace{\left[\sum_{i=0}^{k} \lambda_{i} d(i) \boldsymbol{x}(i)\right]}_{\boldsymbol{p}(k)}
$$

where $\boldsymbol{R}(k)=\boldsymbol{R}(k-1)+\lambda_{k} \boldsymbol{x}(k) \boldsymbol{x}^{T}(k)$ and $\boldsymbol{p}(k)=\boldsymbol{p}(k-$ $1)+\lambda_{k} d(k) \boldsymbol{x}(k)$. 
This means that the BEACON algorithm minimizes the following objective function $\xi(k)$

$$
\xi(k)=\sum_{i=0}^{k} \lambda_{i} \varepsilon^{2}(i)=\boldsymbol{e}^{T}(k) \boldsymbol{e}(k)=\|\boldsymbol{e}(k)\|^{2}
$$

where

$$
\boldsymbol{e}(k)=\boldsymbol{d}(k)-\boldsymbol{X}(k) \boldsymbol{w}(k)
$$

with $\boldsymbol{d}(k)$ being the weighted desired or reference signal vector and $\boldsymbol{X}(k)$ the input data matrix, defined as follows.

$$
\begin{gathered}
\boldsymbol{d}(k)=\left[\begin{array}{c}
\sqrt{\lambda_{k}} d(k) \\
\sqrt{\lambda_{k-1}} d(k-1) \\
\vdots \\
\sqrt{\lambda_{0}} d(0)
\end{array}\right] \\
\boldsymbol{X}(k)=\left[\begin{array}{c}
\sqrt{\lambda_{k}} \boldsymbol{x}^{T}(k) \\
\sqrt{\lambda_{k-1}} \boldsymbol{x}^{T}(k-1) \\
\vdots \\
\sqrt{\lambda_{0}} \boldsymbol{x}^{T}(0)
\end{array}\right]
\end{gathered}
$$

The premultiplication of (8) by the orthogonal matrix (representing an overall triangularization process via elementary Givens rotations matrices) $\boldsymbol{Q}(k)$ triangularizes $\boldsymbol{X}(k)$ without affecting the cost function.

$$
\boldsymbol{Q}(k) \boldsymbol{e}(k)=\left[\begin{array}{l}
\boldsymbol{e}_{q_{1}}(k) \\
\boldsymbol{e}_{q_{2}}(k)
\end{array}\right]=\left[\begin{array}{l}
\boldsymbol{d}_{q_{1}}(k) \\
\boldsymbol{d}_{q_{2}}(k)
\end{array}\right]-\left[\begin{array}{c}
\boldsymbol{O} \\
\boldsymbol{U}(k)
\end{array}\right] \boldsymbol{w}(k)
$$

where $\boldsymbol{U}(k)$ is the Cholesky factor of $\boldsymbol{X}^{T}(k) \boldsymbol{X}(k)$, i.e., product $\boldsymbol{U}^{T}(k) \boldsymbol{U}(k)$ corresponds to $\boldsymbol{X}^{T}(k) \boldsymbol{X}(k)$, and the subscripts 1 and 2 indicate the first $k-N$ and the last $N+1$ components of the vector, respectively.

The weighted-square error (or cost function) can be minimized by choosing $\boldsymbol{w}(k)$ such that the term $\boldsymbol{d}_{q_{2}}(k)-\boldsymbol{U}(k) \boldsymbol{w}(k)$ is zero. The tap-weight coefficients, for the case of a direct QRD-WLS BEACON algorithm (the QRD-based algorithm that updates $\boldsymbol{U}(k)$ from $\boldsymbol{U}(k-1)$ as seen in the following), could be computed using the well-known back-substitution procedure.

Using the fact that $\boldsymbol{Q}(k)$ is orthogonal and the definition of $\boldsymbol{X}(k)$, we can write the product $\boldsymbol{Q}(k) \boldsymbol{X}(k)$ as

$\boldsymbol{Q}(k) \underbrace{\left[\begin{array}{cc}1 & \mathbf{0}^{T} \\ \mathbf{0} & \boldsymbol{Q}^{T}(k-1)\end{array}\right]\left[\begin{array}{cc}1 & \mathbf{0}^{T} \\ \mathbf{0} & \boldsymbol{Q}(k-1)\end{array}\right]}_{\boldsymbol{I}} \underbrace{\left[\begin{array}{c}\sqrt{\lambda_{k} \boldsymbol{x}^{T}(k)} \\ \boldsymbol{X}(k-1)\end{array}\right]}_{\boldsymbol{X}(k)}=\left[\begin{array}{c}\boldsymbol{O} \\ \boldsymbol{U}(k)\end{array}\right]$

such that the following fixed order expression to update the (here assumed lower triangular matrix) Cholesky factor is obtained.

$$
\left[\begin{array}{c}
\mathbf{0}^{T} \\
\boldsymbol{U}(k)
\end{array}\right]=\boldsymbol{Q}_{\theta}(k)\left[\begin{array}{c}
\sqrt{\lambda_{k}} \boldsymbol{x}^{T}(k) \\
\boldsymbol{U}(k-1)
\end{array}\right]
$$

The last expression shows the update of the Cholesky factor $\boldsymbol{U}(k)$. Matrix $\boldsymbol{Q}_{\theta}(k)$ can be partitioned as

$$
\boldsymbol{Q}_{\theta}(k)=\left[\begin{array}{cc}
\gamma(k) & \boldsymbol{g}^{T}(k) \\
\boldsymbol{f}(k) & \boldsymbol{E}(k)
\end{array}\right]
$$

where:
$\gamma(k)=\prod_{i=1}^{N} \cos \theta_{i}(k), \theta_{i}(k)$ are the rotation angles in $\boldsymbol{Q}_{\theta}(k)$; $\boldsymbol{f}(k)=\sqrt{\lambda_{k}} \boldsymbol{U}^{-T}(k) \boldsymbol{x}(k)$; $\boldsymbol{E}(k)=\boldsymbol{U}^{-T}(k) \boldsymbol{U}^{T}(k-1)$; $\boldsymbol{g}(k)=-\gamma(k) \boldsymbol{a}(k)=-\gamma(k) \sqrt{\lambda_{k}} \underbrace{\boldsymbol{U}^{-T}(k-1) \boldsymbol{x}(k)}_{\overline{\boldsymbol{a}}(k)}$.

The Inverse QRD-RLS algorithm [7], instead, updates the inverse of the Cholesky factor. In order to derive the inverse QRD-RLS algorithm we start from the basic update equation of the deterministic weighted autocorrelation matrix and write it in terms of the Cholesky factor matrix.

$$
\mathbf{U}^{T}(k) \mathbf{U}(k)=\mathbf{U}^{T}(k-1) \mathbf{U}(k-1)+\lambda_{k} \mathbf{x}(k) \mathbf{x}^{T}(k)
$$

Taking the inverse of both sides and using the matrix inversion lemma $\left(A+[B C D]^{-1}=A^{-1}-A^{-1} B\left[B A^{-1} D+\right.\right.$ $\left.\left.C^{-1}\right]^{-1} D A^{-1}\right)$, the update for the inverse Cholesky factor is obtained.

$$
\begin{aligned}
& \mathbf{U}^{-1}(k) \mathbf{U}^{-T}(k)=\mathbf{U}^{-1}(k-1) \mathbf{U}^{-T}(k-1) \\
& -\frac{\sqrt{\lambda_{k}} \mathbf{U}^{-1}(k-1) \mathbf{U}^{-T}(k-1) \mathbf{x}(k) \mathbf{x}^{T}(k) \mathbf{U}^{-1}(k-1) \mathbf{U}^{-T}(k-1) \sqrt{\lambda_{k}}}{\mathbf{x}^{T}(k) \mathbf{U}^{-1}(k-1) \sqrt{\lambda_{k}} \sqrt{\lambda_{k}} \mathbf{U}^{-T}(k-1) \mathbf{x}(k)+1}
\end{aligned}
$$

Using the definition of $\mathbf{a}(k)$, defining $\boldsymbol{u}(k)=$ $-\gamma(k) \sqrt{\lambda_{k}} \boldsymbol{U}^{-1}(k-1) \boldsymbol{U}^{-T}(k-1) \boldsymbol{x}(k)$, and $\gamma(k)=$ $\frac{1}{\sqrt{1+\mathbf{a}^{T}(k) \mathbf{a}(k)}}$, the update equation becomes

$$
\mathbf{U}^{-1}(k) \mathbf{U}^{-T}(k)=\mathbf{U}^{-1}(k-1) \mathbf{U}^{-T}(k-1)-\mathbf{u}(k) \mathbf{u}^{T}(k)
$$

The updating equation for the new algorithm, the IQRDWLS BEACON, is obtained following from the QRD-RLS expression and $\mathrm{Eq}(17)$ :

$$
\left[\begin{array}{c}
\boldsymbol{u}^{T}(k) \\
\boldsymbol{U}^{-T}(k)
\end{array}\right]=\boldsymbol{Q}_{\theta}(k)\left[\begin{array}{c}
\mathbf{0}^{T} \\
\boldsymbol{U}^{-T}(k-1)
\end{array}\right]
$$

It is observed that with the updating of $\boldsymbol{U}^{-T}(k)$ we can compute vector $\boldsymbol{a}(k)$, and that from $\boldsymbol{a}(k)$ we can obtain matrix $\boldsymbol{Q}_{\theta}(k)$.

In order to have all necessary equations, the vector updating equation is obtained from the BEACON algorithm by realizing that vector $\boldsymbol{\kappa}(k)$ corresponds to ${ }^{1}-\sqrt{\lambda_{k}} \gamma(k) \boldsymbol{u}(k)$ :

$$
\boldsymbol{w}(k)=\boldsymbol{w}(k-1)-e(k) \gamma(k) \sqrt{\lambda_{k}} \boldsymbol{u}(k) .
$$

The new algorithm is detailed in Table II.

\section{Simulation RESUlts}

In this section, we present the results of an experiment carried out in order to show the performance of the proposed algorithm in a system identification scenario. We used the IQRD-WLS BEACON algorithm to identify an unknown plant $w_{O P T}(k)=\delta(k)+0.9 \delta(k-1)-0.8 \delta(k-2)+0.1 \delta(k-3)+$ $0.6 \delta(k-4)+0.2 \delta(k-5)-0.4 \delta(k-6)+0.2 \delta(k-7)-0.1 \delta(k-8)$. We have used $N=9$ (no undermodeling) and a colored input signal produced by passing Gaussian white noise through an IIR filter with system function given by $\frac{1}{1+1.2 z^{-1}+0.81 z^{-2}}$ and normalizing its variance (such that $\sigma_{x}^{2}=1$ ). The observation noise was white noise with $\sigma_{n}^{2}$ such that the $S N R$ was set

\footnotetext{
${ }^{1}$ Noting that $\boldsymbol{S}(k-1)=\boldsymbol{R}^{-1}(k-1)=\boldsymbol{U}^{-1}(k-1) \boldsymbol{U}^{-T}(k-1)$, we replace it in the definition of vector $\boldsymbol{\kappa}(k)$, and simplify.
} 
TABEL II

THE NEW ALGORITHM.

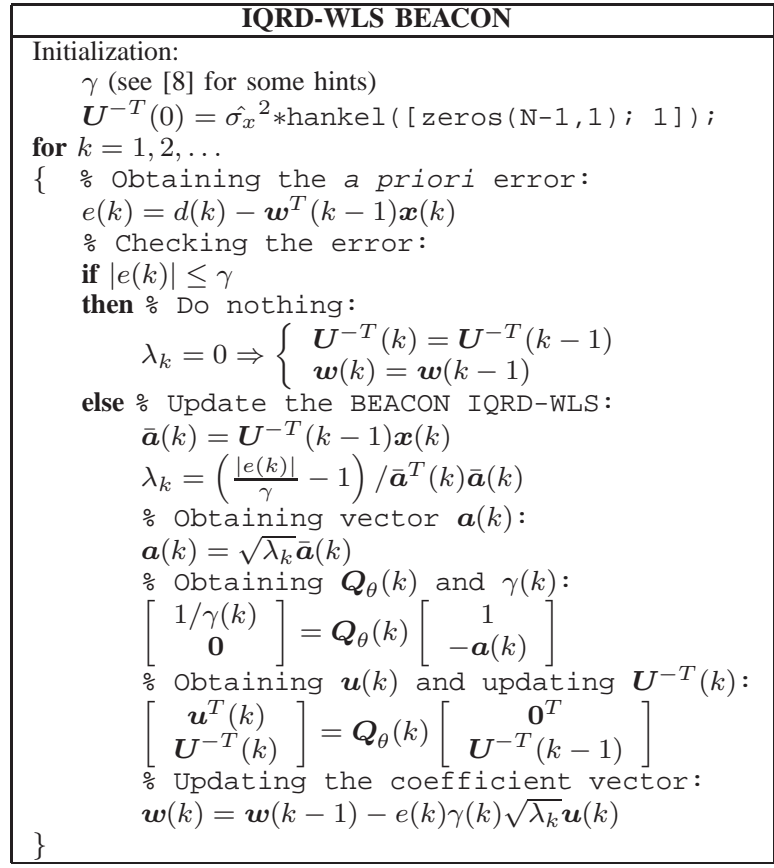

to $80 \mathrm{~dB}$. In this experiment, we used a noise threshold $\gamma=$ $\sqrt{4 \sigma_{n}^{2}}$ and the results were averaged over 10,000 independent runs.

The learning curve of the proposed algorithm, identical to the BEACON algorithm also implemented, is depicted in Fig. 2. The conventional RLS algorithm and the IQRDRLS algorithm, with a forgetting factor $\lambda=0.99$, were also implemented for comparison and, as expected, also presented identical learning curves. It is worth mentioning that identical curves are obtained only if (a careful) equivalent initialization is observed. We can note in this figure that the BEACON algorithm outperforms the RLS algorithms in terms of speed of convergence; nevertheless, a slightly higher misadjustment is the price for this better performance. As any other setmembership algorithm, the BEACON can trade off rate of updating with misadjustment. Certainly, a smaller value of $\gamma$ would cause a lower misadjustment with the cost of an increase in the number of updates. In this experiment, the proposed algorithm was updated approximately $13.6 \%$ of the iterations.

\section{CONCLUSions}

In this paper, we have proposed a new algorithm corresponding to the Inverse QRD version of the bounding ellipsoidal adaptive constrained least-squares (BEACON) algorithm. The proposed algorithm, once minimizing the same objective function and assumed properly initialized, presents an identical learning curve when compared to the conventional BEACON algorithm.

All expressions for this new algorithm were derived and resulted to be coherent with the case of non updating; this is so because, for this case, $\lambda_{k}=0$ and the main variable of the algorithm keeps unaltered, $\boldsymbol{U}^{-T}(k)=\boldsymbol{U}^{-T}(k-1)$. This is



Fig. 2. Learning curves of BEACON and RLS algorithms.

important since it allows the existence of this version; the same does not occur when one attempts to derive a fast $(\mathcal{O}(\mathcal{N})$ ) version for this algorithm. The main difficulty for obtaining a fast version of the proposed algorithm raises from the fact that the input data matrix as seen in (12) no longer presents a shift structure as in the case of the QRD-RLS algorithms. Further investigation is required to obtain a Fast QRD-WLS BEACON algorithm as well as to investigate the stability of the proposed algorithm compared to the conventional BEACON algorithm, that is, to check if the innovation check and the time varying lambda of the new algorithm has (or not) altered the attractive numerical properties of the original Givens rotation based Inverse QRD-RLS algorithm.

\section{ACKNOWLEDGMENTS}

This work was partially funded by CNPq (Brazil) and the Academy of Finland, SMARAD CoE (Finland).

\section{REFERENCES}

[1] S. Gollamudi, S. Nagaraj, S. Kapoor, and Y. F. Huang, "Set-membership filtering and a set-membership normalized LMS algorithm with an adaptive step size," IEEE Signal Processing Letters, vol. 5, no. 5, pp. 111-114, May 1998.

[2] S. Werner and P. S. R. Diniz, "Set-membership affine projection algorithm," IEEE Signal Processing Letters, vol. 8, no. 8, pp. 231-235, Aug. 2005.

[3] J. R. Deller, Jr., "Set membershio identification in digital signal processing," IEEE ASSP Magazine, vol. 6, no. 4, pp. 4-20, Oct. 1989.

[4] S. Nagaraj, S. Gollamudi, S. Kapoor, and Y.-F. Huang, "BEACON: An Adaptive Set-Membership Filtering Technique with Sparse Updates," IEEE Transactions on Signal Processing, Vol. 47, no. 11, pp. 29282941, Nov. 1999.

[5] J. R. Deller, Jr. and S. F. Odeh, "Adaptive Set-Membership Identification in $\mathcal{O}(m)$ Time for Linear-in-Parameters Models," IEEE Transactions on Signal Processing, vol. 41, no. 5, pp. 1906-1924, May 1993.

[6] P. S. R. Diniz, Adaptive Filtering: Algorithms and Practical Inplementations. Kluwer Academic Publishers, 2nd edition 2002.

[7] S. T. Alexander and A. L. Ghirnikar, "A method for recursive least squares adaptive filtering based upon an inverse QR decomposition," IEEE Transactions on Signal Processing, vol. SP-41, no. 1, pp. 20-30, January 1993.

[8] J. F. Galdino, J. A. A. Jr., and M. L. R. de Campos, "A set-membership NLMS algorithm with time-varying error bound," in Proc. Int. Symp. Circuits and Systems (ISCAS), Island of Kos, Greece, May 2006. 